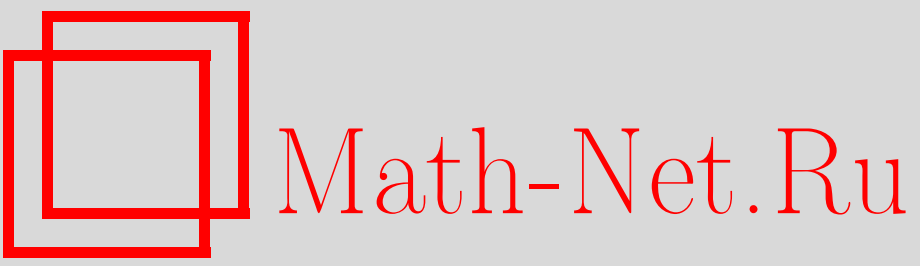

И. Брийе, С. Гонера, П. Косински, П. Масланка, С. Гиллер, Квадратичные интегралы движения для систем тождественных частиц в квантовом случае, ТМФ, 2005, том 144, номер 2, 290-294

DOI: https://doi.org/10.4213/tmf1853

Использование Общероссийского математического портала Math-Net.Ru подразумевает, что вы прочитали и согласны с пользовательским соглашением

http://www.mathnet.ru/rus/agreement

Параметры загрузки:

IP : 3.89.185.249

26 апреля 2023 г., 14:18:50 
ТЕОРЕТИЧЕСКАЯ

И МАТЕМАТИЧЕСКАЯ

ФИЗИКА

Том 144, № 2

август, 2005

(C) 2005 г.

И. Брийе*, С. Гонера ${ }^{\dagger}$, П. Косински ${ }^{\dagger}$,

П. Масланка ${ }^{\dagger}$, С. Гиллер ${ }^{\ddagger}$

\section{КВАДРАТИЧНЫЕ ИНТЕГРАЛЫ ДВИЖЕНИЯ ДЛЯ СИСТЕМ ТОЖДЕСТВЕННЫХ ЧАСТИЦ В КВАНТОВОМ СЛУЧАЕ}

Рассмотрены квантовые динамические системы тождественных частиц, допускающих дополнительный интеграл движения, квадратичный по импульсам. Обнаружено, что существует подходящий способ упорядочения, который позволяет превратить классические интегралы движения в их квантовые аналоги. Рассмотрена связь этих интегралов с разделением переменных в уравнении Шредингера.

Ключевые слова: квантовые интегралы движения, модели Калоджеро-Сазерленда-Мозера, разделение переменных, симметрия $\operatorname{sl}(2, \mathbb{R})$.

\section{1. ВВЕДЕНИЕ}

В работе [1] Браден показал, что перестановочная симметрия налагает жесткие ограничения на вид потенциалов, допускающих полиномиальные по импульсам интегралы движения. Точнее, он доказал, что только система, допускающая интеграл движения третьего порядка с трансляционно-инвариантным старшим членом, является знаменитой моделью Калоджеро-Сазерленда-Мозера.

В предыдущей работе [2] мы исследовали классические системы тождественных частищ, обладающие квадратичными интегралами движения. Была получена полная классификация таких систем. В настоящей работе рассматриваются их квантовые модификации.

\section{2. КЛАССИЧЕСКИЙ СЛУЧАЙ}

Приведем основной результат работы [2]. Предположим, что выполнены следуюшие условия:

\footnotetext{
*Faculte des Sciences, Universite de Mons, 7000 Mons, Belgium

${ }^{\dagger}$ Department of Theoretical Physics II University of Łódź, Pomorska 149/153, 90-236 Łódź, Poland. E-mail: cgonera@uni.lodz.pl; pmaslan@uni.lodz.pl

${ }^{\ddagger}$ Pedagogical University of Czestochowa, Armii Krajowey 13/15, 42-200 Czestochowa, Poland
} 
1) гамильтониан $H$ имеет естественный вид:

$$
H=\frac{1}{2} \sum_{i=1}^{N} p_{i}^{2}+V\left(q_{1}, \ldots, q_{N}\right) ;
$$

2) гамильтониан $H$ является трансляционно-инвариантным:

$$
\{H, P\}=0, \quad P=\sum_{i=1}^{N} p_{i} ;
$$

3) гамильтониан $H$ инвариантен относительно действия группы $S_{N}$ всех перестановок канонических переменных $q_{i}, p_{i}, i=1, \ldots, N$;

4) гамильтониан $H$ допускает сушествование по меньшей мере одного интеграла движения, квадратичного по импульсам и функционально не зависящего от $H$ и $P$.

Тогда общий вид потенциала $V$ есть

$$
V(\underline{q})=\widetilde{V}(\underline{q})+U\left(\sum_{i, j=1}^{N}\left(q_{i}-q_{j}\right)^{2}\right),
$$

где $\widetilde{V}$ - трансляционно-инвариантная однородная функция степени 2 , а $U$-произвольная дифференцируемая функция одной переменной.

Вид дополнительных интегралов зависит от потенциала. Если $U \equiv 0$ (или, в более общем случае, $U \equiv$ const), любой квадратичньй интеграл является линейной комбинацией следуюших интегралов:

$$
\begin{aligned}
& I_{1}=\left(\sum_{k=1}^{N} q_{k} p_{k}\right) P-2 Q H \\
& I_{2}=2\left(\sum_{k=1}^{N} q_{k}^{2}\right) H-\left(\sum_{k=1}^{N} q_{k} p_{k}\right)^{2}, \\
& I_{3}=2 Q P\left(\sum_{k=1}^{N} q_{k} p_{k}\right)-2 Q^{2} H-\left(\sum_{k=1}^{N} q_{k}^{2}\right) P^{2},
\end{aligned}
$$

где $Q=\sum_{k=1}^{N} q_{k}$. Интегралы $I_{1}, I_{2}, I_{3}$ удовлетворяют соотношению

$$
I_{1}^{2}+P^{2} I_{2}+2 H I_{3}=0
$$

В случае нетривиальной функции $U$ сушествует один интеграл

$$
\begin{aligned}
I_{4}= & 2\left(N\left(\sum_{k=1}^{N} q_{k}^{2}\right)-Q^{2}\right) \widetilde{H}-N\left(\sum_{k=1}^{N} q_{k} p_{k}\right)^{2}+ \\
& +2 Q P\left(\sum_{k=1}^{N} q_{k} p_{k}\right)-P^{2}\left(\sum_{k=1}^{N} q_{k}^{2}\right)
\end{aligned}
$$

где $\widetilde{H}$ получается из $H$ заменой $V \rightarrow \widetilde{V}$.

Мы показали, что приведенные вьше интегралы связаны с разделением переменных в уравнении Гамильтона-Якоби. Точнее, они возникают в результате отделения радиальных переменных в координатах Якоби. Кроме того, в работах [3] объяснено, как эти интегралы связаны с конформной симметрией $s l(2, \mathbb{R})$. 


\section{3. КВАНТОВЫЙ СЛУЧАЙ}

Как видно из формул (4), (6), выражения для интегралов не могут быть прямо перенесены на квантовый случай, так как возникает проблема упорядочения. Однако, поскольку гамильтониан имеет естественный вид, можно ожидать, что связанные с разделением переменных в уравнении Гамильтона-Якоби интегралы возникнут в квантовом случае как постоянные разделения переменных в уравнении Шредингера.

Предполагая, что потенциал имеет вид (3), методом проб и ошибок можно найти правильный способ упорядочения для квантовых аналогов интегралов $I_{1}-I_{4}$ :

$$
\begin{aligned}
I_{1}= & \frac{1}{4}\left(\sum_{k=1}^{N}\left(q_{k} p_{k}+p_{k} q_{k}\right)\right) P+\frac{1}{4} P\left(\sum_{k=1}^{N}\left(q_{k} p_{k}+p_{k} q_{k}\right)\right)-Q H-H Q \\
I_{2}= & \left(\sum_{k=1}^{N} q_{k}^{2}\right) H+H\left(\sum_{k=1}^{N} q_{k}^{2}\right)-\frac{1}{4}\left(\sum_{k=1}^{N}\left(q_{k} p_{k}+p_{k} q_{k}\right)\right)^{2} \\
I_{3}= & \frac{1}{4}\left[\left(\sum_{k=1}^{N}\left(q_{k} p_{k}+p_{k} q_{k}\right)\right)(Q P+P Q)+(Q P+P Q)\left(\sum_{k=1}^{N}\left(q_{k} p_{k}+p_{k} q_{k}\right)\right)\right]- \\
& -Q^{2} H-H Q^{2}-\frac{1}{2}\left(\sum_{k=1}^{N} q_{k}^{2}\right) P^{2}-\frac{1}{2} P^{2}\left(\sum_{k=1}^{N} q_{k}^{2}\right), \\
I_{4}= & \left(N\left(\sum_{k=1}^{N} q_{k}^{2}\right)-Q^{2}\right) \widetilde{H}+\widetilde{H}\left(N\left(\sum_{k=1}^{N} q_{k}^{2}\right)-Q^{2}\right)-\frac{1}{4} N\left(\sum_{k=1}^{N}\left(q_{k} p_{k}+p_{k} q_{k}\right)\right)^{2}+ \\
& +\frac{1}{4}\left[\left(\sum_{k=1}^{N}\left(q_{k} p_{k}+p_{k} q_{k}\right)\right)(Q P+P Q)+(Q P+P Q)\left(\sum_{k=1}^{N}\left(q_{k} p_{k}+p_{k} q_{k}\right)\right)\right]- \\
& -\frac{1}{2}\left(\sum_{k=1}^{N} q_{k}^{2}\right) P^{2}-\frac{1}{2} P^{2}\left(\sum_{k=1}^{N} q_{k}^{2}\right) .
\end{aligned}
$$

Можно проверить непосредственно, что приведенные выше выражения являются эрмитовыми и коммутируют с гамильтонианом (для того чтобы $I_{1}, I_{2}, I_{3}$ были постоянными движения, снова нужно положить $V=\widetilde{V})$.

\section{4. РАЗДЕЛЕНИЕ ПЕРЕМЕННЫХ}

Как и в классическом случае, можно установить связь квадратичных интегралов с разделением переменных; соответствующим уравнением является теперь уравнение Шредингера. Отделим движение центра масс и введем полярные переменные в пространстве координат Якоби. Тогда интеграл $I_{4}$ возникает в результате отделения радиальной переменной. 
Действительно, определим переменные

$$
\begin{aligned}
\tilde{q}_{i} & =q_{i}-\frac{1}{N} Q, \\
\tilde{p}_{i} & =p_{i}-\frac{1}{N} P, \\
\rho & =\sqrt{\sum_{k=1}^{N} \tilde{q}_{k}^{2},} \\
p_{\rho} & =\frac{1}{2} \sum_{k=1}^{N}\left(\frac{\tilde{q}_{k}}{\rho} \tilde{p}_{k}+\tilde{p}_{k} \frac{\tilde{q}_{k}}{\rho}\right) .
\end{aligned}
$$

Тогда гамильтониан может быть записан в виде

$$
H=\frac{1}{2 N} P^{2}+\frac{M^{2}}{2 \rho^{2}}+\frac{1}{2} p_{\rho}^{2}+\frac{\hbar^{2}(N-2)(N-4)}{8 \rho^{2}}+\frac{F}{\rho^{2}}+U\left(\rho^{2}\right),
$$

где $M^{2}$ - квадрат момента импульса в системе центра масс, т.е.

$$
M^{2}=\frac{1}{2} \sum_{i, j=1}^{N}\left(\tilde{q}_{i} \tilde{p}_{j}-\tilde{q}_{j} \tilde{p}_{i}\right)^{2}
$$

Мы также использовали потенциал общего вида $(3)$, вследствие чего функция $F$ зависит только от угловых переменных. Из формулы (9) можно сделать вьвод, что оператор

$$
\rho^{2}\left(\widetilde{H}-\frac{1}{2 N} P^{2}-\frac{1}{2} p_{\rho}^{2}-\frac{\hbar^{2}(N-2)(N-4)}{8 \rho^{2}}\right)
$$

отвечает за отделение радиальной переменной. Теперь можно проверить, что выражение (11) эквивалентно выражению

$$
\frac{1}{2 N} I_{4}-\frac{\hbar^{2}(N-2)(N-4)}{8} .
$$

\section{5. СИММЕТРИЯ $s l(2, \mathbb{R})$}

Полученные для случая $U \equiv 0$ результаты могут быть осмыслены с точки зрения симметрии $\operatorname{sl}(2, \mathbb{R})[3]$. Определяя операторы

$$
X=\sum_{k=1}^{N} q_{k}^{2}, \quad Y=\frac{1}{2} \sum_{k=1}^{N}\left(q_{k} p_{k}+p_{k} q_{k}\right)
$$

легко убедиться, что вместе с оператором $H$ они образуют алгебру $s l(2, \mathbb{R})$ :

$$
[Y, H]=2 i \hbar H, \quad[Y, X]=-2 i \hbar X, \quad[H, X]=-2 i \hbar Y .
$$


Оператор Казимира этой алгебры является, разумеется, интегралом движения. Нетрудно видеть, что он равен $I_{2}$ :

$$
I_{2}=X H+H X-Y^{2} .
$$

Для того чтобы объяснить смысл интеграла $I_{1}$, заметим следующее. Любой интеграл движения $I$, который является однородной функцией натуральной степени $n$, задает вектор старшего веса для некоторого представления в присоединенном действии алгебры $s l(2, \mathbb{R}),[H, I]=0,[Y, I]=i \hbar n I$. Поэтому эволюция ближайшего к старшему вектора $J=(i \hbar)^{-1}[X, I]$ линейна по времени:

$$
\dot{J}=\frac{1}{i \hbar}[J, H]=-2 n I .
$$

Теперь, если $I^{\prime}$ - другой интеграл степени $n^{\prime}$, то из выражения (15) следует, что $n^{\prime} I^{\prime} J-$ $n I J^{\prime}$ также является интегралом движения. Применяя это рассуждение к триплету $(H, Y, X)$ и дублету $(P, Q)$, получаем $I_{1}$.

Благодарности. Работа поддержана грантом KBN № 5Р03В06021.

\section{Список литературы}

[1] H. W. Braden. Rigidity, Functional Equations and the Calogero-Moser Model. solv-int/0005046.

[2] Y. Brihaye, S. Giller, C. Gonera, P. Kosinski, P. Maslanka. Czech. J. Phys. 2004. V. 54. № 11. P. 1185.

[3] P. I. Gambardella. J. Math. Phys. 1975. V. 16. P. 1172; G. Barucchi, T. Regge. J. Math. Phys. 1977. V. 18. P. 1149; S. Wojciechowski. Phys. Lett. A. 1977. V. 64. P. 273; C. Gonera, P. Kosinski. Acta Phys. Pol. B. 1999. V. 30. P. 907; C. Gonera. Phys. Lett. A. 1998. V. 237. P. 365 . 